Sandra Mrozinska1, 2, Tomasz Gosiewski ${ }^{3}$, Agnieszka Sroka-Oleksiak ${ }^{3}$, Magdalena Szopa1, 2 , Małgorzata Bulanda ${ }^{3}$, Maciej T Malecki1,2, Tomasz Klupa ${ }^{1,2}$

${ }^{1}$ Department of Metabolic Diseases, Jagiellonian University Medical College, Krakow, Poland

2University Hospital, Krakow, Poland

${ }^{3}$ Department of Microbiology, Jagiellonian University Medical College, Krakow, Poland

\title{
The effect of linagliptin treatment on gut microbiota in patients with HNF1A-MODY or type 2 diabetes - a preliminary cohort study
}

\section{ABSTRACT}

Introduction. Many studies have evaluated the relationship between diabetes and microbiota. In animal models, the dipeptidyl peptidase-4 inhibitors altered the gut microbiota. We investigated whether linagliptin alters the gastrointestinal flora in humans.

Materials and methods. This prospective cohort study enrolled 24 patients: 5 patients with maturity onset diabetes of the young associated with HNF1A mutation and 19 patients with type 2 diabetes mellitus. Stool samples were collected at baseline and 4 weeks after treatment intensification with either linagliptin or a sulphonylurea alongside current treatment. Faecal 16S rRNA was analysed by next-generation sequencing. Results. Nine patients initiated linagliptin whereas 15 patients initiated or increased the dose of a sulphonylurea. After linagliptin treatment, we did not observe changes in taxa in L2-L7 based on analysis of composition of microbiomes (ANCOM). The same held true for pairwise alpha diversity (Shannon diversity, $p=0.59 ;$ Pielou's measure of evenness, $p=0.68$; and observed operational taxonomic units [OTUs], $p=0.77$ )

Address for correspondence:

prof. dr hab. n. med. Tomasz Klupa

Katedra Chorób Metabolicznych

Uniwersytet Jagielloński w Krakowie

Phone: +48124248301

Fax: +48124219786

e-mail: tomasz_klupa@yahoo.com

Clinical Diabetology 2019, 8, 6, 263-270

DOI: $10.5603 /$ DK.2019.0024

Received: 06.07.2019

Accepted: 01.10.2019 and beta diversity distances (unweighted UniFrac, $p=0.99$; weighted UniFrac, $p=0.93$; Bray-Curtis, $p=0.98$; and Jaccard, $p=0.99$ ). Similarly, after sulphonylurea intensification, we did not observe changes in taxa in L2-L7 in ANCOM, nor were there changes in alpha diversity (Shannon diversity, $p=0.19$; Pielou's measure of evenness, $p=0.21$; and observed OTUs, $p=0.42$ ) or beta diversity distances (unweighted UniFrac, $p=0.99$; weighted UniFrac, $p=0.99$; Bray-Curtis, $p=1 ;$ and Jaccard, $p=0.99$ ).

Conclusion. We did not observe changes in colonic microbiota 4 weeks after addition of linagliptin to current diabetes treatment. Further studies are required to determine whether linagliptin influences the colonic microbiota in humans. (Clin Diabetol 2019; 8, 6: 263-270)

Key words: diabetes, HNF 1 alpha, linagliptin, microflora, sulfonylurea

\section{Introduction}

Despite extensive research in type 2 diabetes (T2DM), the pathogenesis of the condition and the factors underpinning disease progression and therapeutic response remain incompletely understood [1]. Established risk factors for T2DM include obesity, sedentary lifestyle, older age and strong family history of the disease [2]. Genome-wide association studies have provided valuable insights into the genetic predisposition to various conditions, including T2DM, but have had 
limited success in explaining the heritability of complex diseases [3, 4]. To account for this unexplained heritability, a role has been proposed for gene-environment interactions, including gastrointestinal bacterial flora or nutrition/medication-related alterations in gastrointestinal hormone activity [3].

Many recent studies have focused on the relationship between T2DM and gastrointestinal bacterial flora [5]. For example, differences in microbiota have been demonstrated between healthy subjects and patients with monogenic forms of diabetes [6, 7]. Obesity, a risk factor for T2DM, has also been associated with alterations in gut microbiota [8]; however, body mass index-independent differences in bacterial flora have been observed in patients with T2DM compared with healthy subjects [9]. Some changes in microbiota previously shown in patients with T2DM versus healthy individuals have been attributed to metformin use [10].

Dipeptidyl peptidase-4 (DPP-4) inhibitors are a well-established class of medications used widely in the management of T2DM [11]. Of interest, it has been shown that at least three DPP-4 inhibitors (sitagliptin, saxagliptin and vildagliptin) may alter the structure of gastrointestinal flora in animal models [12-16]. Some other antidiabetic medications, including liraglutide and acarbose, are also postulated to modify gut microbiota [12]. However, it remains unclear whether these effects on the microbiota influence therapeutic response.

The DPP-4 inhibitor linagliptin has not been studied in regard to gut microbiota and is the only member of the drug class that is primarily eliminated as the parent compound in faeces (95\%) [17]. The aim of this prospective study was to compare the colonic bacterial flora structure before and after the addition of linagliptin to current treatment in patients with T2DM or maturity onset diabetes of the young associated with HNF1A mutation (HNF1A-MODY).

\section{Materials and methods}

Study setting and eligibility

The cohort study was conducted between 2013 and 2015 at the Department of Metabolic Diseases, Jagiellonian University Medical College, Krakow, Poland and the University Hospital, Krakow, Poland, in collaboration with the Center for Medical Genomics (OMICRON) and Department of Microbiology, JagielIonian University Medical College, Krakow, Poland. Patients treated in our Outpatient Clinic and volunteers took a part in a study. The cohort has been described in detail previously [7]. Briefly, the study enrolled men and women aged 18-65 years with T2DM or HNF1A-MODY receiving metformin alone or metformin plus a sulphonylurea (SU) at or below the submaximal dose, or (for patients with HNF1A-MODY only) insulin therapy. Patients were also required to have poorly controlled glycaemia (glycosylated haemoglobin $\left[\mathrm{HbA}_{1 \mathrm{c}}\right]$ $>7 \%$ or $>53 \mathrm{mmol} / \mathrm{mol}$ ) and no advanced, chronic complications of diabetes. Confirmed patient readiness to cooperate with the research centre was required for study participation. All participants declared not using antibiotics 4 weeks before stool sample collection. The exclusion criteria were as follows: lack of consent to participate in the study, withdrawal during the study, taking antibiotics or probiotics up to 30 days before the sample collection, confirmed infection of the gastrointestinal tract, chronic inflammatory bowel disease of unknown etiology, active cancer (especially of the gastrointestinal tract), immunodeficiency, features of liver damage (with the exception of nonalcoholic fatty liver transaminase levels less than three times the upper limit of the normal level). The study received approval from the Jagiellonian University Ethics Committee. All participants provided written informed consent in accordance with the Declaration of Helsinki.

\section{Laboratory investigations}

Blood samples were obtained from all patients. $\mathrm{HbA}_{1 \mathrm{c}^{\prime}}$ triglycerides, total cholesterol, low-density lipoprotein cholesterol, high-density lipoprotein cholesterol, C-reactive protein, aspartate transaminase and alanine transaminase were assessed by standard laboratory techniques.

\section{Treatment and follow-up}

Diabetes therapy was intensified in all patients. In the linagliptin group, patients were prescribed linagliptin in addition to current treatment, while in the control group (SU group), the dose of a current SU was increased or a new SU was initiated alongside current treatment. Treatment allocation was not randomised, but was instead based on local guidelines and long-term drug affordability for the patient (given the substantially greater cost of DPP-4 inhibitors than SU in Poland). Patients were asked to avoid changing their dietary habits and level of physical activity during the study.

Stool samples were collected from all patients before and 4 weeks after treatment intensification. In 21 patients, information from blinded continuous glucose monitoring (iPro2, Medtronic, Dublin, Ireland) was obtained from 1 week before and 3 weeks after treatment intensification (i.e., in the fourth week of intensified treatment).

RNA isolation and 16S metagenomic sequencing

Bacterial RNA was isolated using Genomic Mini AX Stool Spin (A\&A Biotechnology, Gdynia, Poland), 
modified to include enzymatic treatment (lysozyme, lysostaphin and lyticase) and a bead-beating step. Libraries were prepared according to the Illumina 16S Metagenomic Sequencing Library Preparation protocol (https://support.illumina.com/content/dam/ /illumina-support/documents/documentation/chemistry_documentation/16s/16s-metagenomic-libraryprep-guide-15044223-b.pdf). Briefly, universal external primers were used to amplify regions V3 and V4 of the $16 \mathrm{~S}$ rRNA. After the polymerase chain reaction cleanup, samples were indexed, cleaned and pooled. Finally, 10 pM libraries with $10 \%$ PhiX Spike-In were sequenced on Illumina MiSeq (Illumina, Inc., San Diego, CA, US) using the V3 sequencing kit (300 bp paired-end reads).

\section{Sequencing data analysis}

Samples were processed and analysed using the Quantitative Insights Into Microbial Ecology 2 (QIIME2, version 2018.11) [18] custom pipeline. Briefly, the quality of demultiplexed paired-end reads from MiSeq $(2 \times$ $300 \mathrm{bp}$ ) was evaluated and the reads were trimmed to remove primers and poor-quality bases with cutadapt [19]. Trimmed sequences were denoised and joined with DADA2 [20]. Next, closed-reference clustering of features [21] and reference-based chimera filtering were performed using vsearch [22] and the Greengenes database at 99\% similarity [23]. Generated operational taxonomic units (OTUs) were assigned to taxonomy using a naive Bayes classifier [24], which was pre-trained on the sequenced target RNA regions. For further analysis, we included only features occurring in at least three samples that had more than 20 total reads. The bacterial composition was analysed at phylum, order, family, genus and species levels. Filtered feature tables were used to generate the trees for phylogenetic diversity analyses. Rarefaction curve analysis was used to estimate the completeness of microbial community sampling. We also computed default alpha and beta diversity metrics and generated principal coordinates analysis (PCOA) plots for each of the beta diversity metrics using Emperor [25]. Group significance between alpha and beta diversity indices were calculated with the QIIME2 longitudinal pairwise-differences plugin using the $t$-test. Correlations with alpha diversity indices were calculated with the QIIME2 plugin using Spearman correlation. Differential abundance between groups at each taxonomic level was tested using analysis of composition of microbiomes (ANCOM) [26].

\section{Statistical analysis}

The reported $p$-values were not corrected for multiple testing. Continuous variables are presented as medians and interquartile ranges (IQRs), whereas categorical variables are expressed as counts with percentages. Groups were compared with the MannWhitney $U$ test, Fisher's exact test and Wilcoxon test using Statistica 13 software (StatSoft Inc., Tulsa, OK, US). Post-hoc power analysis was performed in package R [27], v.3.5.1. Power was set at 0.8 and the significance level (alpha level) was set at 0.05 .

\section{Results}

Patient population

A total of 60 patients were initially interviewed, of whom 29 patients with T2DM and 11 patients with HNF1A-MODY agreed to participate and follow the study protocol. Of this group, we enrolled 24 patients who met the eligibility criteria.

Five patients had HNF1A-MODY and 19 patients had T2DM. The median age was 60 years. Median $\mathrm{HbA}_{1 \mathrm{c}}$ was $66 \mathrm{mmol} / \mathrm{mol}(8.2 \%)$, with an IQR of $62-70$ $\mathrm{mmol} / \mathrm{mol}$ (7.8-8.6\%). In 5 patients with T2DM and 4 patients with HNF1A-MODY, linagliptin was added to current treatment (linagliptin group). In 1 patient with HNF1A-MODY and 14 patients with T2DM, an SU was initiated or the dose of a current SU was increased according to local guidelines (SU group). There were no differences in patient characteristics between groups at baseline (Table 1).

\section{S rRNA sequencing}

Sequencing analysis of the 48 samples provided a mean 57,145 reads per sample (median, 52,547). The best sample contained 142,914 read pairs, while the worst contained 10,608 . OTU picking resulted in 570, with total frequency of $2,742,964$ features.

\section{Bacterial profile at baseline}

In the linagliptin and SU groups, respectively, baseline bacterial profiles with an abundance of $>1 \%$ at the phylum level were Actinobacteria $(6.23 \%$ vs. $10.98 \%)$, Bacteroidetes (1.15\% vs. $2.02 \%$ ), Firmicutes $(88.42 \%$ vs. $78.83 \%)$, Proteobacteria (1.83\% vs. $2.81 \%$ ) Verrucomicrobia (2.13\% vs. $5.09 \%$ ) and 'other' $(0.24 \%$ vs. $0.28 \%$; Figure $1 \mathrm{~A})$. At the class level, baseline bacterial profiles included Actinobacteria (1.27\% vs. $4.50 \%)$, Coriobacteriia (4.96\% vs. $6.47 \%)$, Bacteroidia $(1.15 \%$ vs. $2.02 \%)$, Bacilli $(2.21 \%$ vs. $2.39 \%)$, Clostridia (81.12\% vs. 71.89\%), Erysipelotrichi (5.09\% vs. $4.55 \%$ ), Gammaproteobacteria (1.56\% vs. $2.75 \%$ ), Verrucomicrobiae (2.13\% vs. 5.09\%) and 'other' $(0.50 \%$ vs. $0.33 \%$; Figure $1 \mathrm{~B})$.

Comparing the SU group with the linagliptin group prior to intensification, we observed a single significantly increased abundance at the class level (for Actinobacteria; centred log-ratio $[\mathrm{clr}]=2.57, \mathrm{~W}=5$ ). 
Table 1. Patient characteristics at baseline

\begin{tabular}{|c|c|c|c|c|}
\hline Variable* & $\begin{array}{l}\text { All patients } \\
\qquad(n=24)\end{array}$ & $\begin{array}{l}\text { Linagliptin group } \\
\qquad(n=9)\end{array}$ & $\begin{array}{l}\text { SU group } \\
(n=15)\end{array}$ & p-value \\
\hline Male sex, n (\%) & $13(54.2)$ & $5(55.6)$ & $8(53.3)$ & 0.42 \\
\hline Age (years) & $60(55.5-62.5)$ & $55(50-61)$ & $60(57-63)$ & 0.16 \\
\hline Duration of diabetes (years) & $5(1.9-9)$ & $11(1.3-23)$ & $5(2.5-6)$ & 0.24 \\
\hline $\mathrm{BMI}\left[\mathrm{kg} / \mathrm{m}^{2}\right]$ & $29.7(26.8-32.2)$ & $29(26.8-32)$ & $30(26.8-32.5)$ & 0.73 \\
\hline Obesity, n (\%) & $12(50.0)$ & $3(33.3)$ & $9(60.0)$ & 0.99 \\
\hline $\mathrm{HbA}_{1 \mathrm{c}}(\%)$ & $8.2(7.8-8.6)$ & $8.4(8-9.7)$ & $8.2(7.6-8.4)$ & 0.11 \\
\hline Creatinine $[\mu \mathrm{mol} / \mathrm{L}]$ & $81.2(69.6-92)$ & $75(69.1-82)$ & $85(76.2-99.2)$ & 0.1 \\
\hline $\mathrm{TG}[\mathrm{mmol} / \mathrm{L}]$ & $2.4(1.4-2.9)$ & $2.9(1.6-3.1)$ & $1.9(1.2-2.6)$ & 0.14 \\
\hline $\mathrm{TC}[\mathrm{mmol} / \mathrm{L}]$ & $5.4(4.4-6.1)$ & $5.2(4.8-6.2)$ & $5.5(4.2-5.9)$ & 0.77 \\
\hline LDL-C [mmol/L] & $3.1(2.3-3.8)$ & $2.3(1.9-3.7)$ & $3.2(2.4-3.8)$ & 0.24 \\
\hline $\mathrm{HDL}-\mathrm{C}[\mathrm{mmol} / \mathrm{L}]$ & $1.2(1.1-1.4)$ & $1.2(1.1-1.3)$ & $1.2(1-1.4)$ & 0.82 \\
\hline $\mathrm{CRP}[\mathrm{mg} / \mathrm{L}]$ & $3.3(1-6.6)$ & $1.1(0.7-4)$ & $4.2(1.5-7.5)$ & 0.06 \\
\hline AST [IU/L] & $21.5(18.5-29)$ & $19(18-33)$ & $23(20-26)$ & 0.73 \\
\hline ALT $[I U / L]$ & $37(27-48)$ & $38(36-45)$ & $34(25-49)$ & 0.48 \\
\hline Leucocytes $\left[\times 10^{9} / \mathrm{L}\right]$ & $6.9(6-8.7)$ & $7.2(6.3-9.9)$ & $6.5(5.8-8.6)$ & 0.24 \\
\hline Erythrocytes $\left[\times 10^{9} / L\right]$ & $4.8(4.6-5.1)$ & $5(4.9-5.2)$ & $4.6(4.5-5.1)$ & 0.11 \\
\hline Haemoglobin $[\mathrm{g} / \mathrm{dL}]$ & $14.7(13.7-15.6)$ & $14.9(14.7-15.9)$ & $14(13.5-15)$ & 0.06 \\
\hline Haematocrit (\%) & $43.7(41-45.3)$ & $44.1(43.8-45.9)$ & $42.3(40.5-44.6)$ & 0.08 \\
\hline Platelets $\left[\times 10^{9} / \mathrm{L}\right]$ & $220.5(196.5-253)$ & $222(187-231)$ & $219(197-274)$ & 0.52 \\
\hline
\end{tabular}

*Continuous variables are summarised as medians (IQRs), qualitative variables are presented as the number (percentages)

ALT - alanine transaminase; AST — aspartate transaminase; BMI — body mass index; CRP - C-reactive protein; DPP-4 - dipeptidyl peptidase-4;

$\mathrm{HbA}_{1 \mathrm{c}}$ - glycosylated haemoglobin; HDL-C — high-density lipoprotein cholesterol; IQR — interquartile range; LDL-C — low-density lipoprotein cholesterol;

SU — sulfonylurea; TC — total cholesterol; TG — triglycerides

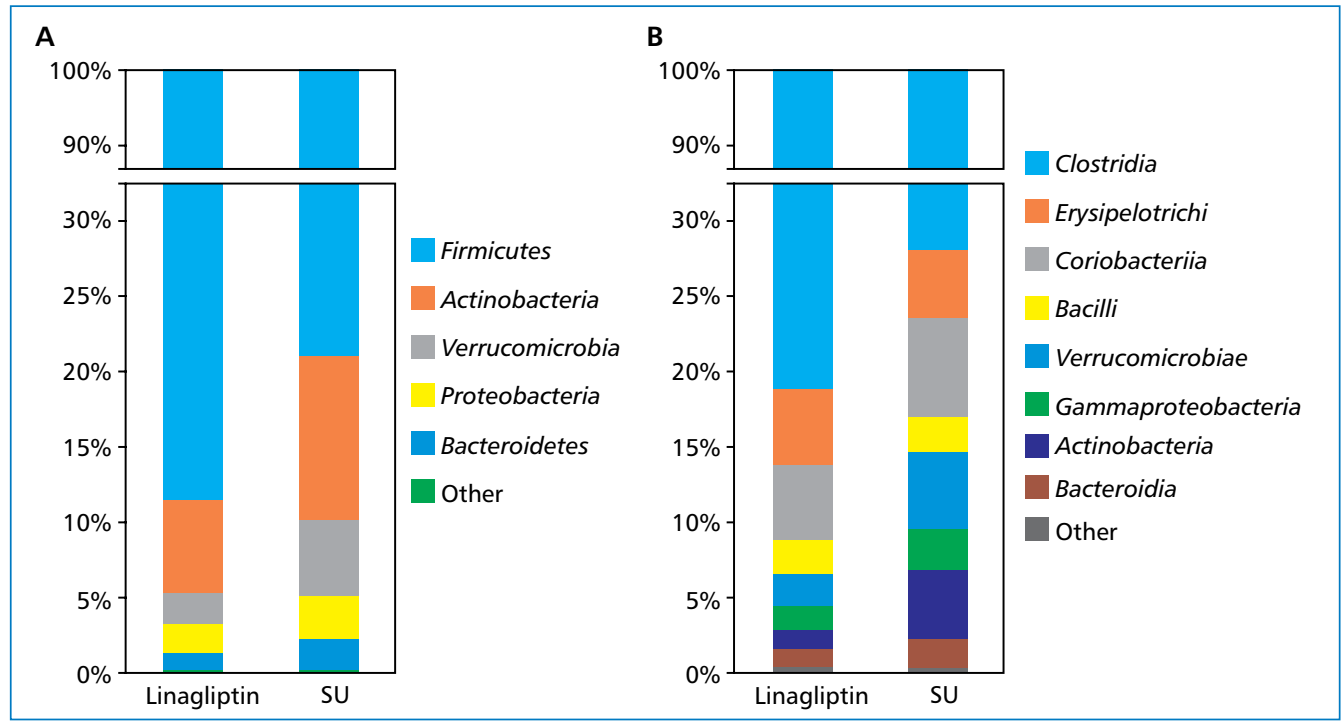

Figure 1. Composition of the bacterial community at the phylum - L2 (A) and class - L3 (B) levels for linagliptin and SU patients prior to intensification. Taxa with abundance below $1 \%$ were merged and represented as 'other'

No significant differences were observed between groups in ANCOM results for phylum, order, family, genus or species at baseline.

No differences in within-sample phylotype richness and evenness (alpha diversity) metrics were detected between groups. Shannon diversity $(p=0.14)$, Pielou's measure of evenness $(p=0.16)$, observed OTUs $(p=0.10)$ and Faith's phylogenetic diversity $(p=0.18)$ did not differ between linagliptin and SU samples (Figure 2). Linagliptin and SU samples did not show statisti- 


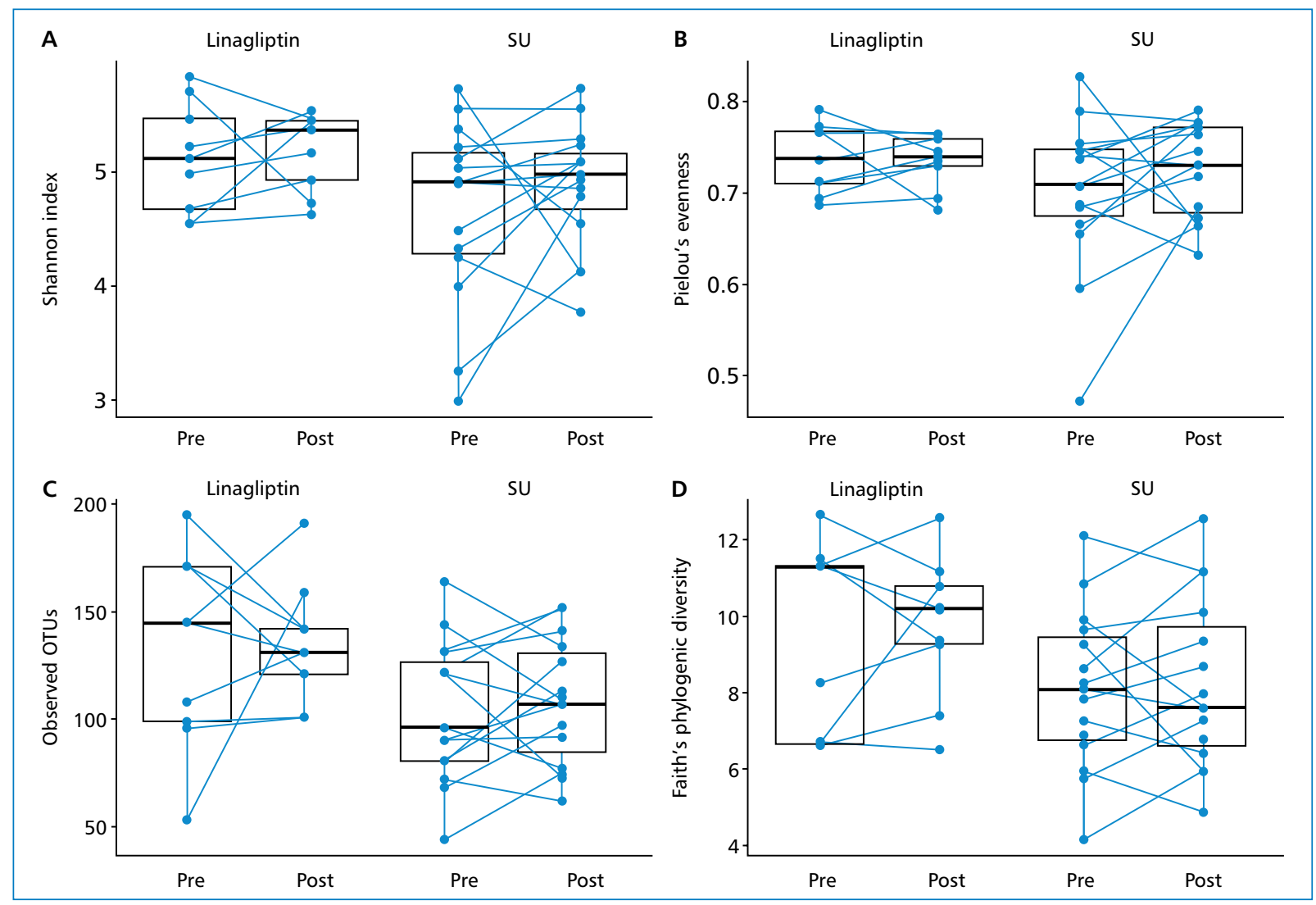

Figure 2. Pairwise alpha diversity analysis of linagliptin and sulfonylurea (SU) groups before and after treatment intensification. Differences in alpha diversity were measured by Shannon index (A), Pielou's measure of species evenness (B), observed OTUs (C) and Faith's phylogenetic diversity (D). Student's $t$-test was performed to analyse statistical significance

cally differences in any evaluated distance metrics: unweighted UniFrac ( $p=0.39$ ), weighted UniFrac $(p=0.60)$, Bray-Curtis $(p=0.78)$ and Jaccard $(p=0.39)$. Moreover linagliptin and SU patients were not separated or clustered according to PCoA of beta diversity metrics (Figure 3).

The two groups shared 397 OTUs, whereas 77 OTUs were unique to the linagliptin group and 89 were unique to the SU group.

\section{Bacterial profile after optimisation of treatment}

After 4 weeks of linagliptin treatment, ANCOM results showed no changes in taxa in L2-L7 compared with baseline. The same held true for pairwise alpha diversity, where differences in Shannon diversity $(p=0.59)$, Pielou's measure of evenness $(p=0.68)$, observed OTUs ( $p=0.77$ ) and Faith's phylogenetic diversity ( $p=0.51$ ) were not statistically significant (Figure 2). Moreover, after treatment intensification, unweighted UniFrac ( $p=0.99$, weighted UniFrac $(p=0.93)$, Bray-Curtis $(p=0.98)$ and Jaccard $(p=0.99)$ distances were also not significant (Figure 3 ). After treatment with linagliptin, we observed 43 new OTUs while 58 OTUs present at baseline were lost.

After optimisation of therapy in the SU group, we did not observe changes in taxa in L2-L7, compared with baseline, in the ANCOM analysis. We did not demonstrate changes in alpha diversity metrics (Shannon diversity, $p=0.19$; Pielou's measure of evenness, $p=0.21$; observed OTUs, $\mathrm{p}=0.42$; and Faith's phylogenetic diversity, $p=0.65$; Figure 2 ) or beta diversity distances (unweighted UniFrac, $\mathrm{p}=0.99$; weighted UniFrac, $p=0.99$; Bray-Curtis, $p=0.99$; and Jaccard, $p=0.99$; Figure 3). After treatment, we observed 50 new OTUs while 37 OTUs present at baseline were lost.

\section{Continuous glucose monitoring}

Continuous glucose monitoring data were collected before and after optimisation of treatment in 21 patients. The median glucose level in the linagliptin group $(n=9)$ was 178 (171-190) $\mathrm{mg} / \mathrm{dL}$ at baseline and 166 (157-174) $\mathrm{mg} / \mathrm{dL}$ after intensification $(\mathrm{p}=0.02)$. In patients in the SU group with available data $(n=12)$, the median glucose level was 168 (159.5-190.5) mg/dL at baseline and 144.5 (131.5-154) $\mathrm{mg} / \mathrm{dL}$ at follow-up ( $\mathrm{p}=0.015)$. 


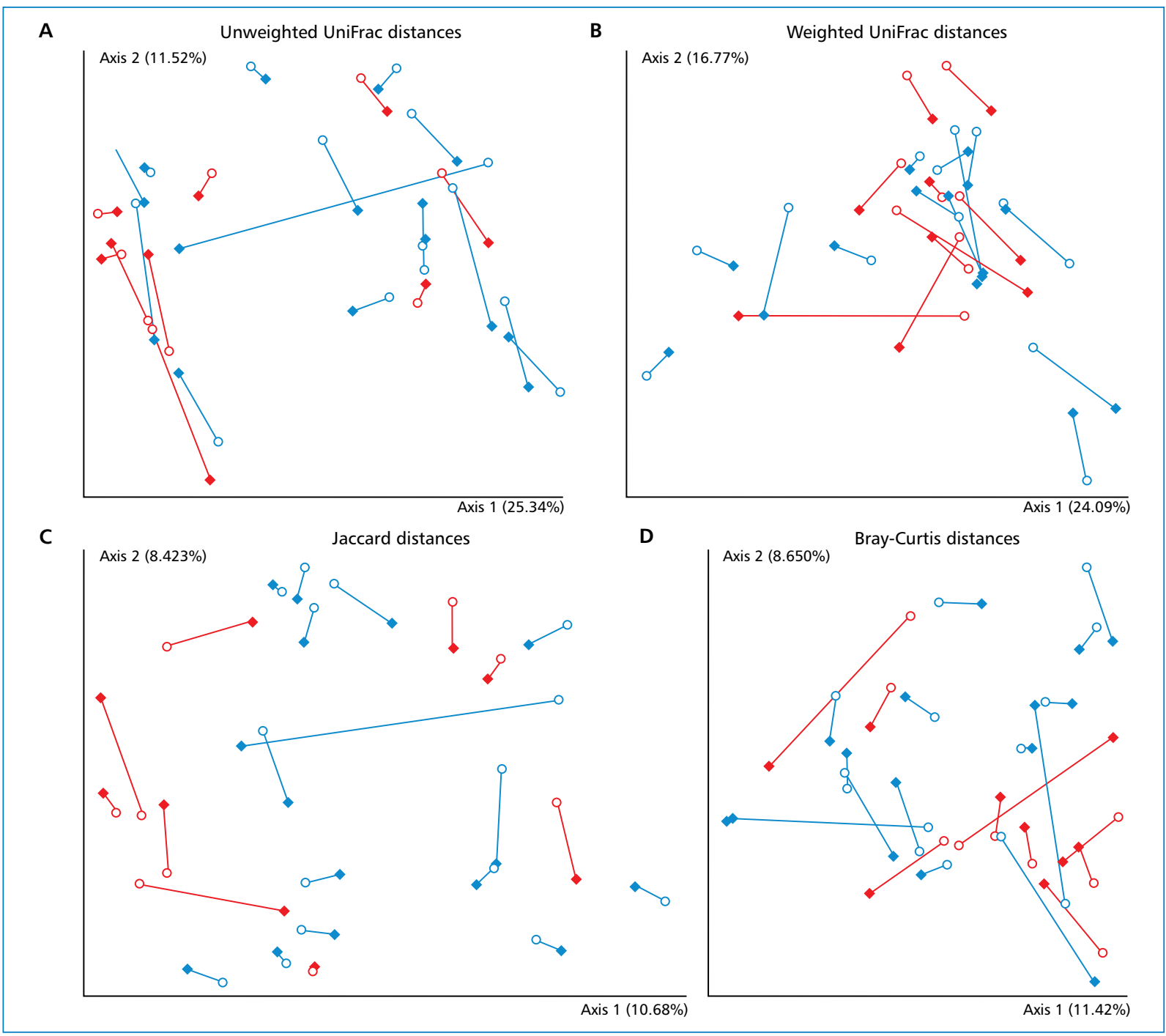

Figure 3. PCoA 2D plots of beta diversity analysis of linagliptin and sulfonylurea (SU) patients before and after treatment intensification. Beta diversity was measured by unweighted UniFrac distances (A), weighted UniFrac distances (B), Jaccard distances (C) and Bray-Curtis distances (D). Permutational multivariate analysis of variance (PERMANOVA) was performed to analyse statistical significance. Paired samples are connected with lines. Red lines represent linagliptin group and blue lines represent SU group. Rhombus represent pre-treatment intensification samples and rings represent post-treatment intensification samples

\section{Post-hoc power analysis (sample size calculation)}

Based on our results, we determined that comparing the SU group with the linagliptin group before intensification, we need at least 43 samples per group to achieve 0.8 statistical power with the 0.05 significance level. As for pre and post-treatment samples, the necessary sample size to achieve desired power at desired significance level is at least 95 patients for the linagliptin group, and at least 53 patients for SU group.

\section{Discussion}

To our knowledge, this is the first study to compare microbiota before and after the addition of linagliptin to current treatment in patients with T2DM or HNF1A-MODY. Although the analysis failed to show differences in microbiotal composition after treatment with either the DPP-4 inhibitor or SU intensification, our study was performed on a very small number of patients, in linagliptin group there were only nine patients.

The study was underpowered. No statistical methods were used to predetermine sample size and the final number of patients was limited. Post-hoc analysis revealed that much larger groups and need to detected differences between group.

The small sample size was at least partially due to the limitations on patients and requirement for stool samples imposed by the study protocol, which may have discouraged patient participation. In addition, due to expected difficulties with longer-term patient compliance, the study was designed to have a relatively 
short follow-up period (4 weeks). We cannot exclude that differences in microbiotal composition would be observed during longer follow-up. Further studies should be conducted to examine and confirm this hypothesis-generating study. Of note, previous animal studies using DPP-4 inhibitors reported some changes in colonic microbiota after 8-12 weeks, although the results were equivocal [13-16].

So far, the effects of three DPP-4 inhibitors (vildagliptin, sitagliptin and saxagliptin) on microbiota have been investigated in animal models [13-16], but there are no previous animal or human studies on the effects of alogliptin or linagliptin on gut microbiota. Olivares et al. reported a reduction in genus Oscillibacter spp. and unclassified Ruminococcaceae (OTU 241) following 8 weeks of vildagliptin use [13]. Zhang et al. also observed changes in bacterial flora after administration of vildagliptin in diabetic rats for 12 weeks [14]. Yan et al. showed changes in microbiota composition in diabetic rats after the administration of sitagliptin for 12 weeks, with an increase in the relative abundance of Bacteroidetes and Proteobacteria, and a decrease in Firmicutes after treatment [15]. Zhang et al. also reported a higher relative abundance of Bacteroidetes and lower abundance of Firmicutes after treatment with vildagliptin. Both sitagliptin [15] and vidagliptin [14] have been shown to increase the amount of butyrateproducing bacteria. Finally, Wang et al. observed an increase in the relative abundance of Firmicutes after administration of saxagliptin for 8 weeks [16], in contrast to the studies of Yan et al. (sitagliptin) [15] and Zhang et al. (vildagliptin) [14].

One cannot exclude that the lack of effect on colonic microbiota in our study was due the use of linagliptin, which has not yet been evaluated for effects on colonic microflora in animals. The observed effect of linagliptin on microbiota across patients in our study was heterogeneous, however, we failed to determine factors associated with this phenomenon, possible due to limited patients number in subgroups. In more numerous subgroups we could notice different trends, for example in HNF1A and T2DM patients. Further studies could reveal potential features or phenotypes responsible for different trends in individual patients.

Furthermore, although patients were encouraged to follow general dietary recommendations for diabetes before the study, our results could also have been influenced by individual variations in diet composition, food preparation methods or quality of ingredients. For instance, one could speculate that individuals who could afford linagliptin (which is not reimbursed) could also afford better quality food. It has been reported that microbiota composition is predominately modified by diet $[12,28,29]$. In mice, changes in diet account for $57 \%$ of the variation in gut microbiota and genetic factors for $12 \%$ [30]. One cannot exclude that changes in diet composition attenuated the effect of DPP-4 inhibition.

\section{Conclusions}

We did not observe major differences in the structure and composition of the colonic bacterial flora after treatment with linagliptin in our preliminary study. However, the study was underpowered, and due to the small study size and short follow-up period, we cannot exclude some effect of linagliptin on the colonic microbiota. Further longitudinal studies based on larger populations should be performed to determine whether incretin-based drugs, including DPP-4 inhibitors, influence the colonic bacterial flora.

\section{Acknowledgments}

$16 \mathrm{~S}$ rRNA sequencing and sequencing data analysis were performed at the Center for Medical Genomics (OMICRON), Jagiellonian University Medical College (Krakow, Poland).

\section{Funding}

The project entitled "The influence of the dipeptidyl peptidase- 4 inhibitors on the quantitative and qualitative analyses of the intestinal bacterial flora in patients with type 2 diabetes and in patients with HNF1A diabetes" was co-financed by the European Union from the European Regional Development Fund.

\section{Statement of competing interests}

$S$. Mrozinska received a travel grant form Boehringer Ingelheim and a course grant from EGIS. The remaining authors declared no conflict of interest.

\section{REFERENCES}

1. Kahn SE, Cooper ME, Del Prato S. Pathophysiology and treatment of type 2 diabetes: perspectives on the past, present, and future. Lancet. 2014; 383(9922): 1068-1083, doi: 10.1016/S01406736(13)62154-6, indexed in Pubmed: 24315620.

2. Fletcher B, Gulanick M, Lamendola C. Risk factors for type 2 diabetes mellitus. J Cardiovasc Nurs. 2002; 16(2): 17-23, indexed in Pubmed: 11800065.

3. Manolio TA, Collins FS, Cox NJ, et al. Finding the missing heritability of complex diseases. Nature. 2009; 461(7265): 747-753, doi: 10.1038/nature08494, indexed in Pubmed: 19812666.

4. McCarthy MI. Genetics of T2DM in 2016: Biological and translational insights from T2DM genetics. Nat Rev Endocrinol. 2017; 13(2): 71-72, doi: 10.1038/nrendo.2016.212, indexed in Pubmed: 28051116.

5. Brunkwall L, Orho-Melander M. The gut microbiome as a target for prevention and treatment of hyperglycaemia in type 2 diabetes: from current human evidence to future possibilities. Diabetologia. 2017; 60(6): 943-951, doi: 10.1007/s00125-0174278-3, indexed in Pubmed: 28434033. 
6. Leiva-Gea I, Sánchez-Alcoholado L, Martín-Tejedor B, et al. Gut Microbiota Differs in Composition and Functionality Between Children With Type 1 Diabetes and MODY2 and Healthy Control Subjects: A Case-Control Study. Diabetes Care. 2018; 41(11): 2385-2395, doi: 10.2337/dc18-0253, indexed in Pubmed: 30224347.

7. Mrozinska S, Radkowski P, Gosiewski T, et al. Qualitative Parameters of the Colonic Flora in Patients with HNF1A-MODY Are Different from Those Observed in Type 2 Diabetes Mellitus. J Diabetes Res. 2016; 2016: 3876764, doi: 10.1155/2016/3876764, indexed in Pubmed: 27807544.

8. Vrieze A, Holleman F, Zoetendal EG, et al. The environment within: how gut microbiota may influence metabolism and body composition. Diabetologia. 2010; 53(4): 606-613, doi: 10.1007/ s00125-010-1662-7, indexed in Pubmed: 20101384.

9. Larsen N, Vogensen FK, van den Berg FWJ, et al. Gut microbiota in human adults with type 2 diabetes differs from non-diabetic adults. PLoS One. 2010; 5(2): e9085, doi: 10.1371/journal. pone.0009085, indexed in Pubmed: 20140211.

10. Forslund $K$, Hildebrand $F$, Nielsen $T$, et al. MetaHIT consortium, MetaHIT consortium. Disentangling type 2 diabetes and metformin treatment signatures in the human gut microbiota. Nature. 2015; 528(7581): 262-266, doi: 10.1038/nature15766, indexed in Pubmed: 26633628.

11. Liu X, Xiao Q, Zhang Li, et al. The long-term efficacy and safety of DPP-IV inhibitors monotherapy and in combination with metfor$\min$ in 18,980 patients with type-2 diabetes mellitus - a metaanalysis. Pharmacoepidemiol Drug Saf. 2014; 23(7): 687-698, doi: 10.1002/pds.3586, indexed in Pubmed: 24639059.

12. Montandon SA, Jornayvaz FR. Effects of antidiabetic drugs on gut microbiota composition. Genes (Basel). 2017; 8(10): 250, doi: 10.3390/genes8100250, indexed in Pubmed: 28973971.

13. Olivares M, Neyrinck AM, Pötgens SA, et al. The DPP-4 inhibitor vildagliptin impacts the gut microbiota and prevents disruption of intestinal homeostasis induced by a Western diet in mice. Diabetologia. 2018; 61(8): 1838-1848, doi: 10.1007/s00125018-4647-6, indexed in Pubmed: 29797022.

14. Zhang Q, Xiao X, Li M, et al. Vildagliptin increases butyrateproducing bacteria in the gut of diabetic rats. PLoS One. 2017; 12(10): e0184735, doi: 10.1371/journal.pone.0184735, indexed in Pubmed: 29036231.

15. Yan X, Feng Bo, Li $P$, et al. Microflora Disturbance during Progression of Glucose Intolerance and Effect of Sitagliptin: An Animal Study. J Diabetes Res. 2016; 2016: 2093171, doi: 10.1155/2016/2093171, indexed in Pubmed: 27631013.

16. Wang L, Li P, Tang Z, et al. Structural modulation of the gut microbiota and the relationship with body weight: compared evaluation of liraglutide and saxagliptin treatment. Sci Rep. 2016; 6: 33251, doi: 10.1038/srep33251, indexed in Pubmed: 27633081.

17. Graefe-Mody U, Retlich S, Friedrich C. Clinical pharmacokinetics and pharmacodynamics of linagliptin. Clin Pharmacokinet. 2012;
51(7): 411-427, doi: 10.2165/11630900-000000000-00000, indexed in Pubmed: 22568694.

18. Bolyen E, Rideout JR, Dillon MR, et al. Reproducible, interactive, scalable and extensible microbiome data science using QIIME 2. Nat Biotechnol. 2019; 37(8): 852-857, doi: 10.1038/s41587-0190209-9, indexed in Pubmed: 31341288.

19. Martin M. Cutadapt removes adapter sequences from high-throughput sequencing reads. EMBnet.journal. 2011; 17(1): 10, doi: 10.14806/ej.17.1.200.

20. Callahan BJ, McMurdie PJ, Rosen MJ, et al. DADA2: High-resolution sample inference from Illumina amplicon data. Nat Methods. 2016; 13(7): 581-583, doi: 10.1038/nmeth.3869, indexed in Pubmed: 27214047.

21. Rideout JR, He Y, Navas-Molina JA, et al. Subsampled openreference clustering creates consistent, comprehensive OTU definitions and scales to billions of sequences. PeerJ. 2014; 2 : e545, doi: 10.7717/peerj.545, indexed in Pubmed: 25177538.

22. Rognes T, Flouri $T$, Nichols $B$, et al. VSEARCH: a versatile open source tool for metagenomics. PeerJ. 2016; 4: e2584, doi: 10.7717/peerj.2584, indexed in Pubmed: 27781170.

23. McDonald D, Price MN, Goodrich J, et al. An improved Greengenes taxonomy with explicit ranks for ecological and evolutionary analyses of bacteria and archaea. ISME J. 2012; 6(3): 610-618, doi: 10.1038/ismej.2011.139, indexed in Pubmed: 22134646.

24. Bokulich NA, Kaehler BD, Rideout JR, et al. Optimizing taxonomic classification of marker-gene amplicon sequences with QIIME 2's q2-feature-classifier plugin. Microbiome. 2018; 6(1): 90, doi: 10.1186/s40168-018-0470-z, indexed in Pubmed: 29773078.

25. Vázquez-Baeza Y, Pirrung M, Gonzalez A, et al. EMPeror: a tool for visualizing high-throughput microbial community data. Gigascience. 2013; 2(1): 16, doi: 10.1186/2047-217X-2-16, indexed in Pubmed: 24280061.

26. Mandal S, Van Treuren W, White RA, et al. Analysis of composition of microbiomes: a novel method for studying microbial composition. Microb Ecol Health Dis. 2015; 26: 27663, doi: 10.3402/mehd. v26.27663, indexed in Pubmed: 26028277.

27. R Development Core Team. R: A language and environment for statistical computing. R Foundation for Statistical Computing, Vienna, Austria 2008. URL http://www.R-project.org/.

28. Claesson MJ, Jeffery IB, Conde S, et al. Gut microbiota composition correlates with diet and health in the elderly. Nature. 2012; 488(7410): 178-184, doi: 10.1038/nature11319, indexed in Pubmed: 22797518.

29. Baothman OA, Zamzami MA, Taher I, et al. The role of gut microbiota in the development of obesity and diabetes. Lipids Health Dis. 2016; 15: 108, doi: 10.1186/s12944-016-0278-4, indexed in Pubmed: 27317359.

30. Zhang $C$, Zhang M, Wang $S$, et al. Interactions between gut microbiota, host genetics and diet relevant to development of metabolic syndromes in mice. ISME J. 2010; 4(2): 232-241, doi: 10.1038/ismej.2009.112, indexed in Pubmed: 19865183. 